\title{
Thin accretion disks in stationary axisymmetric wormhole spacetimes
}

\author{
Tiberiu Harkd * \\ Department of Physics and Center for Theoretical and Computational Physics, \\ The University of Hong Kong, Pok Fu Lam Road, Hong Kong \\ Zoltán Kováç卂 \\ Max-Planck-Institut für Radioastronomie, Auf dem Hügel 69, 53121 Bonn, Germany and \\ Department of Experimental Physics, University of Szeged, Dóm Tér 9, Szeged 6720, Hungary \\ Francisco S. N. Lobd团 \\ Centro de Astronomia e Astrofísica da Universidade de Lisboa, \\ Campo Grande, Ed. C8 1749-016 Lisboa, Portugal
}

(Dated: October 25, 2018)

\begin{abstract}
In this paper, we study the physical properties and the equilibrium thermal radiation emission characteristics of matter forming thin accretion disks in stationary axially symmetric wormhole spacetimes. The thin disk models are constructed by taking different values of the wormhole's angular velocity, and the time averaged energy flux, the disk temperature and the emission spectra of the accretion disks are obtained. Comparing the mass accretion in a rotating wormhole geometry with the one of a Kerr black hole, we verify that the intensity of the flux emerging from the disk surface is greater for wormholes than for rotating black holes with the same geometrical mass and accretion rate. We also present the conversion efficiency of the accreting mass into radiation, and show that the rotating wormholes provide a much more efficient engine for the transformation of the accreting mass into radiation than the Kerr black holes. Therefore specific signatures appear in the electromagnetic spectrum of thin disks around rotating wormholes, thus leading to the possibility of distinguishing wormhole geometries by using astrophysical observations of the emission spectra from accretion disks.

PACS numbers: $04.50 . \mathrm{Kd}, 04.70 . \mathrm{Bw}, 97.10 . \mathrm{Gz}$
\end{abstract}

\section{INTRODUCTION}

In a recent paper, the physical properties and characteristics of matter forming thin accretion disks in static and spherically symmetric wormhole spacetimes were extensively analyzed [1]. In particular, the time averaged energy flux, the disk temperature and the emission spectra of the accretion disks were obtained for these exotic geometries, and compared with the Schwarzschild solution. It was shown that more energy is emitted from the disk in a wormhole geometry than in the case of the Schwarzschild potential. These effects in the disk radiation were confirmed in the radial profiles of temperature corresponding to the flux distributions, and in the emission spectrum of the accretion disks. Thus, specific signatures appear in the electromagnetic spectrum, leading to the possibility of distinguishing these static and spherically symmetric wormhole geometries by using astrophysical observations of the emission spectra from accretion disks. The results of [1] further extended the analysis of the emissivity properties of accretion disks around general relativistic compact objects [2, 3, 4, 5, 6, 7].

In the context of stationary axisymmetric spacetimes,

\footnotetext{
*Electronic address: harko@hkucc.hku.hk

$\dagger$ Electronic address: zkovacs@mpifr-bonn.mpg.de

${ }^{\ddagger}$ Electronic address: flobo@cosmo.fis.fc.ul.pt
}

the mass accretion around rotating black holes was studied in general relativity for the first time in [8]. By using an equatorial approximation to the stationary and axisymmetric spacetime of rotating black holes, steadystate thin disk models were constructed, extending the theory of nonrelativistic accretion [9]. In these models hydrodynamical equilibrium is maintained by efficient cooling mechanisms via radiation transport, and the accreting matter has a Keplerian rotation. The radiation emitted by the disk surface was also studied under the assumption that black body radiation would emerge from the disk in thermodynamical equilibrium. The radiation properties of the thin accretion disks were further analyzed in 10, 11], where the effects of the photon capture by the black hole on the spin evolution were presented as well. In these works the efficiency with which black holes convert rest mass into outgoing radiation in the accretion process was also computed.

It is of interest to consider the properties of thin accretion disks around wormhole geometries, as these exotic spacetimes violate the null energy condition (NEC) 1]. Indeed, a wide variety of wormhole solutions have been considered in the literature (we refer the reader to [12, 13] and to [13] for a recent review). It will also prove interesting to further extend the analysis carried out in 1] to stationary axisymmetric wormhole geometries [14], which are physically more realistic objects than their static and spherically symmetric counterparts. It is also important to compare the properties of the thin ac- 
cretion disks around rotating wormholes with the properties of thin disks around rotating black holes. From this comparison it follows that the intensity of the flux emerging from the disk surface is greater for wormholes than for the Kerr black holes with the same geometrical mass and accretion rate. This gives an effective observational method to discriminate between wormholes and black hole type compact general relativistic objects.

The present paper is organized as follows. In Sec. I, we review the formalism and the physical properties of the thin disk accretion onto compact objects, for stationary axisymmetric spacetimes. In Sec. [II] we analyze the basic properties of matter forming a thin accretion disk in rotating wormhole spacetimes. We discuss and conclude our results in Sec. IV] In the Appendix, we present for self-completeness and self-consistency the effective potential for the Kerr black hole. Throughout this work, we use a system of units so that $c=G=\hbar=k_{B}=1$, where $k_{B}$ is Boltzmann's constant.

\section{THERMAL EQUILIBRIUM RADIATION PROPERTIES OF THIN ACCRETION DISKS IN STATIONARY AXISYMMETRIC SPACETIMES}

\section{A. Stationary and axially symmetric spacetimes}

The physical properties and the electromagnetic radiation characteristics of particles moving in circular orbits around general relativistic bodies are determined by the geometry of the spacetime around the compact object. For a stationary and axially symmetric geometry the metric is given in a general form by

$$
d s^{2}=g_{t t} d t^{2}+2 g_{t \phi} d t d \phi+g_{r r} d r^{2}+g_{\theta \theta} d \theta^{2}+g_{\phi \phi} d \phi^{2} .
$$

In the equatorial approximation, which is the case of interest for our analysis, the metric functions $g_{t t}, g_{t \phi}, g_{r r}$, $g_{\theta \theta}$ and $g_{\phi \phi}$ only depend on the radial coordinate $r$, i.e., $|\theta-\pi / 2| \ll 1$.

To compute the relevant physical quantities of thin accretion disks, we determine first the radial dependence of the angular velocity $\Omega$, of the specific energy $\widetilde{E}$, and of the specific angular momentum $\widetilde{L}$ of particles moving in circular orbits in a stationary and axially symmetric geometry through the geodesic equations. The latter take the following form

$$
\begin{aligned}
\frac{d t}{d \tau} & =\frac{\widetilde{E} g_{\phi \phi}+\widetilde{L} g_{t \phi}}{g_{t \phi}^{2}-g_{t t} g_{\phi \phi}} \\
\frac{d \phi}{d \tau} & =-\frac{\widetilde{E} g_{t \phi}+\widetilde{L} g_{t t}}{g_{t \phi}^{2}-g_{t t} g_{\phi \phi}} \\
g_{r r}\left(\frac{d r}{d \tau}\right)^{2} & =-1+\frac{\widetilde{E}^{2} g_{\phi \phi}+2 \widetilde{E} \widetilde{L} g_{t \phi}+\widetilde{L}^{2} g_{t t}}{g_{t \phi}^{2}-g_{t t} g_{\phi \phi}}
\end{aligned}
$$

From Eq. (4) one can introduce an effective potential term as

$$
V_{e f f}(r)=-1+\frac{\widetilde{E}^{2} g_{\phi \phi}+2 \widetilde{E} \widetilde{L} g_{t \phi}+\widetilde{L}^{2} g_{t t}}{g_{t \phi}^{2}-g_{t t} g_{\phi \phi}} .
$$

For stable circular orbits in the equatorial plane the following conditions must hold: $V_{\text {eff }}(r)=0$ and $V_{\text {eff,r }}(r)=0$, where the comma in the subscript denotes a derivative with respect to the radial coordinate $r$. These conditions provide the specific energy, the specific angular momentum and the angular velocity of particles moving in circular orbits for the case of spinning general relativistic compact spheres, given by

$$
\begin{aligned}
\widetilde{E} & =-\frac{g_{t t}+g_{t \phi} \Omega}{\sqrt{-g_{t t}-2 g_{t \phi} \Omega-g_{\phi \phi} \Omega^{2}}}, \\
\widetilde{L} & =\frac{g_{t \phi}+g_{\phi \phi} \Omega}{\sqrt{-g_{t t}-2 g_{t \phi} \Omega-g_{\phi \phi} \Omega^{2}}}, \\
\Omega & =\frac{d \phi}{d t}=\frac{-g_{t \phi, r}+\sqrt{\left(g_{t \phi, r}\right)^{2}-g_{t t, r} g_{\phi \phi, r}}}{g_{\phi \phi, r}} .
\end{aligned}
$$

The marginally stable orbit around the central object can be determined from the condition $V_{\text {eff, } r r}(r)=0$. To this effect, we formally represent the effective potential as

$$
V_{e f f}(r) \equiv-1+\frac{f}{g},
$$

where

$$
\begin{aligned}
f & \equiv \widetilde{E}^{2} g_{\phi \phi}+2 \widetilde{E} \widetilde{L} g_{t \phi}+\widetilde{L}^{2} g_{\phi \phi}, \\
g & \equiv g_{t \phi}^{2}-g_{t t} g_{\phi \phi},
\end{aligned}
$$

and where the condition $g \neq 0$ is imposed. From $V_{\text {eff }}(r)=0$, we obtain first $f=g$. The condition $V_{e f f, r}(r)=0$ provides $f_{, r} g-f g_{, r}=0$. Thus, from these conditions one readily derives $V_{e f f, r r}(r)=0$, which provides the following important relationship

$$
\begin{aligned}
0= & \left(g_{t \phi}^{2}-g_{t t} g_{\phi \phi}\right) V_{e f f, r r} \\
= & \widetilde{E}^{2} g_{\phi \phi, r r}+2 \widetilde{E} \widetilde{L} g_{t \phi, r r}+\widetilde{L}^{2} g_{t t, r r} \\
& -\left(g_{t \phi}^{2}-g_{t t} g_{\phi \phi}\right)_{, r r},
\end{aligned}
$$

where $g_{t \phi}^{2}-g_{t t} g_{\phi \phi}$ (appearing as a cofactor in the metric determinant) never vanishes. By inserting Eqs. (6)-(8) into Eq. (9) and solving this equation for $r$, we obtain the radii of the marginally stable orbits, once the metric coefficients $g_{t t}, g_{t \phi}$ and $g_{\phi \phi}$ are explicitly given.

\section{B. Physical properties of thin accretion disks}

For a thin accretion disk the vertical size (defined in cylindrical coordinates along the $z$-axis) is negligible, as compared to its horizontal extension (defined along the radial direction $r$ ), i.e., the disk height $H$, equal to the maximum half thickness of the disk, is always 
much smaller than the characteristic radius $R$ of the disk, $H \ll R$. The thin disk is assumed to be in hydrodynamical equilibrium, and the pressure gradient, as well as the vertical entropy gradient, are negligible in the disk. The efficient cooling via the radiation over the disk surface prevents the disk from cumulating the heat generated by stresses and dynamical friction. In turn, this equilibrium causes the disk to stabilize its thin vertical size. The thin disk has an inner edge at the marginally stable orbit of the compact object potential, and the accreting matter has a Keplerian motion in higher orbits.

In steady-state accretion disk models, the mass accretion rate $\dot{M}_{0}$ is assumed to be a constant that does not change with time. The physical quantities describing the orbiting matter are averaged over a characteristic time scale, e.g. $\Delta t$, for a total period of the orbits, over the azimuthal angle $\Delta \phi=2 \pi$, and over the height $H[8,9,10]$.

The particles moving in Keplerian orbits around the compact object with a rotational velocity $\Omega=d \phi / d t$ have a specific energy $\widetilde{E}$ and a specific angular momentum $\widetilde{L}$, which in the steady-state thin disk model depend only on the radii of the orbits. These particles, orbiting with the four-velocity $u^{\mu}$, form a disk of an averaged surface density $\Sigma$, the vertically integrated average of the rest mass density $\rho_{0}$ of the plasma. The accreting matter in the disk is modeled by an anisotropic fluid source, where the density $\rho_{0}$, the energy flow vector $q^{\mu}$ and the stress tensor $t^{\mu \nu}$ are measured in the averaged rest-frame (the specific heat was neglected). Then, the disk structure can be characterized by the surface density of the disk [8, 10]

$$
\Sigma(r)=\int_{-H}^{H}\left\langle\rho_{0}\right\rangle d z
$$

with averaged rest mass density $\left\langle\rho_{0}\right\rangle$ over $\Delta t$ and $2 \pi$ and the torque

$$
W_{\phi}^{r}=\int_{-H}^{H}\left\langle t_{\phi}^{r}\right\rangle d z,
$$

with the averaged component $\left\langle t_{\phi}^{r}\right\rangle$ over $\Delta t$ and $2 \pi$. The time and orbital average of the energy flux vector gives the radiation flux $\mathcal{F}(r)$ over the disk surface as $\mathcal{F}(r)=$ $\left\langle q^{z}\right\rangle$.

The stress-energy tensor is decomposed according to

$$
T^{\mu \nu}=\rho_{0} u^{\mu} u^{\nu}+2 u^{(\mu} q^{\nu)}+t^{\mu \nu},
$$

where $u_{\mu} q^{\mu}=0, u_{\mu} t^{\mu \nu}=0$. The four-vectors of the energy and angular momentum flux are defined by $-E^{\mu} \equiv T_{\nu}^{\mu}(\partial / \partial t)^{\nu}$ and $J^{\mu} \equiv T_{\nu}^{\mu}(\partial / \partial \phi)^{\nu}$, respectively. The structure equations of the thin disk can be derived by integrating the conservation laws of the rest mass, of the energy, and of the angular momentum of the plasma, respectively [8, 10]. From the equation of the rest mass conservation, $\nabla_{\mu}\left(\rho_{0} u^{\mu}\right)=0$, it follows that the time averaged rate of the accretion of the rest mass is independent of the disk radius,

$$
\dot{M}_{0} \equiv-2 \pi r \Sigma u^{r}=\text { constant } .
$$

The conservation law $\nabla_{\mu} E^{\mu}=0$ of the energy has the integral form

$$
\left[\dot{M}_{0} \widetilde{E}-2 \pi r \Omega W_{\phi}^{r}\right]_{, r}=4 \pi r \mathcal{F} \widetilde{E},
$$

which states that the energy transported by the rest mass flow, $\dot{M}_{0} \widetilde{E}$, and the energy transported by the dynamical stresses in the disk, $2 \pi r \Omega W_{\phi}{ }^{r}$, is in balance with the energy radiated away from the surface of the disk, $4 \pi r \mathcal{F} \widetilde{E}$. The law of the angular momentum conservation, $\nabla_{\mu} J^{\mu}=0$, also states the balance of these three forms of the angular momentum transport,

$$
\left[\dot{M}_{0} \widetilde{L}-2 \pi r W_{\phi}^{r}\right]_{, r}=4 \pi r \mathcal{F} \widetilde{L} .
$$

By eliminating $W_{\phi}{ }^{r}$ from Eqs. (14) and (15), and applying the universal energy-angular momentum relation $d E=\Omega d J$ for circular geodesic orbits in the form $\widetilde{E}_{, r}=\Omega \widetilde{L}_{, r}$, the flux $\mathcal{F}$ of the radiant energy over the disk can be expressed in terms of the specific energy, angular momentum and of the angular velocity of the compact sphere [8, 10],

$$
\mathcal{F}(r)=-\frac{\dot{M}_{0}}{4 \pi \sqrt{-g}} \frac{\Omega_{, r}}{(\widetilde{E}-\Omega \widetilde{L})^{2}} \int_{r_{m s}}^{r}(\widetilde{E}-\Omega \widetilde{L}) \widetilde{L}_{, r} d r .
$$

Another important characteristic of the mass accretion process is the efficiency with which the central object converts rest mass into outgoing radiation. This quantity is defined as the ratio of the rate of the radiation of the energy of photons escaping from the disk surface to infinity, and the rate at which mass-energy is transported to the central compact general relativistic object, both measured at infinity [8, 10]. If all the emitted photons can escape to infinity, the efficiency is given in terms of the specific energy measured at the marginally stable orbit $r_{m s}$,

$$
\epsilon=1-\widetilde{E}_{m s} .
$$

For Schwarzschild black holes the efficiency $\epsilon$ is about $6 \%$, whether the photon capture by the black hole is considered, or not. Ignoring the capture of radiation by the hole, $\epsilon$ is found to be $42 \%$ for rapidly rotating black holes, whereas the efficiency is $40 \%$ with photon capture in the Kerr potential [11].

The accreting matter in the steady-state thin disk model is supposed to be in thermodynamical equilibrium. Therefore the radiation emitted by the disk surface can be considered as a perfect black body radiation, where the energy flux is given by $\mathcal{F}(r)=\sigma T^{4}(r)$ ( $\sigma$ is the Stefan-Boltzmann constant), and the observed luminosity $L(\nu)$ has a redshifted black body spectrum [3]:

$$
L(\nu)=4 \pi d^{2} I(\nu)=\frac{8}{\pi} \cos \gamma \int_{r_{i}}^{r_{f}} \int_{0}^{2 \pi} \frac{\nu_{e}^{3} r d \phi d r}{\exp \left(h \nu_{e} / T\right)-1} .
$$

Here $d$ is the distance to the source, $I(\nu)$ is the Planck distribution function, $\gamma$ is the disk inclination angle, and 
$r_{i}$ and $r_{f}$ indicate the position of the inner and outer edge of the disk, respectively. We take $r_{i}=r_{m s}$ and $r_{f} \rightarrow \infty$, since we expect the flux over the disk surface vanishes at $r \rightarrow \infty$ for any kind of general relativistic compact object geometry. The emitted frequency is given by $\nu_{e}=\nu(1+z)$, and the redshift factor can be written as

$$
1+z=\frac{1+\Omega r \sin \phi \sin \gamma}{\sqrt{-g_{t t}-2 \Omega g_{t \phi}-\Omega^{2} g_{\phi \phi}}},
$$

where we have neglected the light bending [15, 16].

The flux and the emission spectrum of the accretion disks around compact objects satisfy some simple scaling relations, with respect to the simple scaling transformation of the radial coordinate, given by $r \rightarrow \widetilde{r}=r / M$, where $M$ is the mass of the compact sphere. Generally, the metric tensor coefficients are invariant with respect of this transformation, while the specific energy, the angular momentum and the angular velocity transform as $\widetilde{E} \rightarrow \widetilde{E}, \widetilde{L} \rightarrow M \widetilde{L}$ and $\Omega \rightarrow \widetilde{\Omega} / M$, respectively. The flux scales as $F(r) \rightarrow F(\widetilde{r}) / M^{4}$, giving the simple transformation law of the temperature as $T(r) \rightarrow T(\widetilde{r}) / M$. By also rescaling the frequency of the emitted radiation as $\nu \rightarrow \widetilde{\nu}=\nu / M$, the luminosity of the disk is given by $L(\nu) \rightarrow L(\widetilde{\nu}) / M$. On the other hand, the flux is proportional to the accretion rate $\dot{M}_{0}$, and therefore an increase in the accretion rate leads to a linear increase in the radiation emission flux from the disk.

\section{ACCRETION DISK PROPERTIES IN ROTATING WORMHOLE GEOMETRIES}

\section{A. Metric and field equations}

The canonical metric for a stationary, axisymmetric traversable wormhole can be written as [14]

$d s^{2}=-N^{2} d t^{2}+e^{\mu} d r^{2}+r^{2} K^{2}\left[d \theta^{2}+\sin ^{2} \theta(d \phi-\omega d t)^{2}\right]$,

where $N, \mu, K$ and $\omega$ are functions of $r$ and $\theta$. To ensure that the metric is nonsingular on the rotation axis $(\theta=0, \pi)$, regularity conditions on $N, \mu$ and $K$ have to be imposed [14], which essentially means that their respective $\theta$ derivatives have to vanish on the rotation axis.

For simplicity, we shall consider the following definitions [14]

$$
N(r, \theta)=e^{\Phi(r, \theta)}, \quad e^{-\mu(r, \theta)}=1-\frac{b(r, \theta)}{r}
$$

which are well suited to describe a traversable wormhole. $\Phi(r, \theta)$ is the redshift function, which needs to be finite to ensure that there are no event horizons or curvature singularities. $b(r, \theta)$ is the shape function which satisfies $b \leq r$ and the flaringout condition. $K(r, \theta)$ determines the proper radial distance, while $\omega$ governs the angular velocity of the wormhole.

The scalar curvature of the space-time (20) is extremely messy, but at the throat $r=r_{0}$ simplifies to

$$
\begin{aligned}
R= & -\frac{1}{r^{2} K^{2}}\left(\mu_{\theta \theta}+\frac{1}{2} \mu_{\theta}^{2}\right)-\frac{\mu_{\theta}}{N r^{2} K^{2}} \frac{(N \sin \theta)_{\theta}}{\sin \theta} \\
& -\frac{2}{N r^{2} K^{2}} \frac{\left(N_{\theta} \sin \theta\right)_{\theta}}{\sin \theta}-\frac{2}{r^{2} K^{3}} \frac{\left(K_{\theta} \sin \theta\right)_{\theta}}{\sin \theta} \\
& +e^{-\mu} \mu_{r}\left[\ln \left(N r^{2} K^{2}\right)\right]_{r}+\frac{\sin ^{2} \theta \omega_{\theta}^{2}}{2 N^{2}} \\
& +\frac{2}{r^{2} K^{4}}\left(K^{2}+K_{\theta}^{2}\right),
\end{aligned}
$$

where the subscripts denote partial derivatives with respect to $r$ and $\theta$. Note that the only troublesome terms are the ones involving $\mu_{\theta}$ and $\mu_{\theta \theta}$, i.e.,

$$
\mu_{\theta}=\frac{b_{\theta}}{(r-b)}, \quad \mu_{\theta \theta}+\frac{1}{2} \mu_{\theta}^{2}=\frac{b_{\theta \theta}}{r-b}+\frac{3}{2} \frac{b_{\theta}^{2}}{(r-b)^{2}} .
$$

Thus, one needs to impose that $b_{\theta}=0$ and $b_{\theta \theta}=0$ at the throat to avoid curvature singularities. This condition shows that the throat is located at a constant value of $r$.

The stress-energy tensor components are extremely complicated, but assume a more simplified form in an orthonormal reference frame and evaluated at the throat. They have the following nonzero components

$$
\begin{aligned}
\left.8 \pi T_{\hat{t} \hat{t}}\right|_{r=r_{0}}= & -\frac{\left(K_{\theta} \sin \theta\right)_{\theta}}{r^{2} K^{3} \sin \theta}-\frac{\omega_{\theta}^{2} \sin ^{2} \theta}{4 N^{2}}+e^{-\mu} \mu_{r} \frac{(r K)_{r}}{r K}+\frac{K^{2}+K_{\theta}^{2}}{r^{2} K^{4}}, \\
\left.8 \pi T_{\hat{r} \hat{r}}\right|_{r=r_{0}}= & \frac{\left(K_{\theta} \sin \theta\right)_{\theta}}{r^{2} K^{3} \sin \theta}-\frac{\omega_{\theta}^{2} \sin ^{2} \theta}{4 N^{2}}+\frac{\left(N_{\theta} \sin \theta\right)_{\theta}}{N r^{2} K^{2} \sin \theta}-\frac{K^{2}+K_{\theta}^{2}}{r^{2} K^{4}}, \\
\left.8 \pi T_{\hat{\theta} \hat{\theta}}\right|_{r=r_{0}}= & \frac{N_{\theta}(K \sin \theta)_{\theta}}{N r^{2} K^{3} \sin \theta}+\frac{\omega_{\theta}^{2} \sin ^{2} \theta}{4 N^{2}}-\frac{\mu_{r} e^{-\mu}(N r K)_{r}}{2 N r K}, \\
8 \pi T_{\left.\hat{\phi} \hat{\phi}\right|_{r=r_{0}}=} & -\frac{\mu_{r} e^{-\mu}(N K r)_{r}}{2 N K r}-\frac{3 \sin ^{2} \theta \omega_{\theta}^{2}}{4 N^{2}}+\frac{N_{\theta \theta}}{N r^{2} K^{2}}-\frac{N_{\theta} K_{\theta}}{N r^{2} K^{3}}, \\
\left.8 \pi T_{\hat{t} \hat{\phi}}\right|_{r=r_{0}}= & \frac{1}{4 N^{2} K^{2} r}\left(6 N K \omega_{\theta} \cos \theta+2 N K \sin \theta \omega_{\theta \theta}\right. \\
& \left.-\mu_{r} e^{-\mu} r^{2} N K^{3} \sin \theta \omega_{r}+4 N \omega_{\theta} \sin \theta K_{\theta}-2 K \sin \theta N_{\theta} \omega_{\theta}\right) .
\end{aligned}
$$


The components $T_{\hat{t} \hat{t}}$ and $T_{\hat{i} \hat{j}}$ have the usual physical interpretations, and in particular, $T_{\hat{t} \hat{\phi}}$ characterizes the rotation of the matter distribution.

Taking into account the stress-energy tensor components above, the NEC at the throat is given by

$$
\begin{aligned}
\left.8 \pi T_{\hat{\mu} \hat{\nu}} k^{\hat{\mu}} k^{\hat{\nu}}\right|_{r=r_{0}}= & \mathrm{e}^{-\mu} \mu_{r} \frac{(r K)_{r}}{r K}-\frac{\omega_{\theta}^{2} \sin ^{2} \theta}{2 N^{2}} \\
& +\frac{\left(N_{\theta} \sin \theta\right)_{\theta}}{(r K)^{2} N \sin \theta} .
\end{aligned}
$$

Rather than reproduce the analysis here, we refer the reader to Ref. [14] for details, where it was shown that the NEC is violated in certain regions, and is satisfied in others. Thus, it is possible for an infalling observer to move around the throat, and avoid the exotic matter supporting the wormhole. However, it is important to emphasize that one cannot avoid the use of exotic matter altogether.

\section{B. Electromagnetic signatures of thin accretion disks in rotating wormhole geometries}

The radial geodesic equation (4) for the metric (20) is given by

$$
\left(1-\frac{b}{r}\right)^{-1}\left(\frac{d r}{d \tau}\right)^{2}=V_{e f f}
$$

Using the relationship $g_{t \phi}^{2}-g_{t t} g_{\phi \phi}=r^{2} K^{2} e^{2 \Phi}$, the effective potential takes the following form

$$
\begin{aligned}
V_{e f f}(r)= & -1+\left[\widetilde{E}^{2} r^{2} K^{2}-2 \widetilde{E} \widetilde{L} r^{2} K^{2} \omega\right. \\
& \left.-\widetilde{L}^{2}\left(e^{2 \Phi}-r^{2} K^{2} \omega^{2}\right)\right] /\left(r^{2} K^{2} e^{2 \Phi}\right)
\end{aligned}
$$

In analogy to the effective potential for a Kerr black hole, provided by Eq. (A4), the above relationships may be rewritten in the following manner

$$
r^{4}\left(\frac{d r}{d \tau}\right)^{2}=V(r)
$$

with $V(r)$ given by

$$
V(r)=r^{4}\left(1-\frac{b}{r}\right) V_{e f f}(r) .
$$

In this work, we consider the specific case of

$$
\omega=\frac{2 J}{r^{3}},
$$

where $J=M^{2} a_{*}$ is the total angular momentum of the wormhole. As we are only interested in the equatorial approximation, i.e., $|\theta-\pi / 2| \ll 1$, we also consider $K=1$ throughout our analysis.
We consider the case of $\Phi=-r_{0} / r$, and the following respective shape functions

$$
b=r_{0}, \quad b(r)=\frac{r_{0}^{2}}{r}, \quad b(r)=\sqrt{r r_{0}},
$$

and

$$
b(r)=r_{0}+\gamma r_{0}\left(1-\frac{r_{0}}{r}\right),
$$

with $0<\gamma<1$.

For simplicity, we also assume the following values for the mass and the spin parameter

$$
\begin{aligned}
& M=0.06776 M_{\odot}(=1000 \mathrm{~cm}) \\
& a_{*}=0.2,0.4,0.6,0.8,1.0
\end{aligned}
$$

respectively.

In Figs. 1 6 we plot the energy flux, the disk temperature and the emission spectra $\nu L(\nu)$ emitted by the accretion disk with a mass accretion rate of $\dot{M}_{0}=10^{-12} M_{\odot} / \mathrm{yr}$ for various wormhole geometries. The form functions we have used are given by Eqs. (35) and (36), respectively. We also present, for the sake of comparison, the properties of the thin disks in the Kerr black hole geometry.

Comparing the energy flux, depicted in Figs. 11 and 2. from the thin disk in a stationary rotating wormhole geometry with the one of a Kerr black hole for $a_{*}<0.8$, we see that the intensity of the flux emerging from the disk surface is at least two orders of magnitude greater for wormholes than for the rotating black hole with the same geometrical mass $r_{0}$ and accretion rate $\dot{M}_{0}$. The flux amplitude exhibits the similar dependence on the spin parameter for both types of the rotating central objects: with the increasing values of $a_{*}$, the maximal values of $\mathcal{F}(r)$ are also increasing. As the central object is rotating faster the flux maxima are shifted closer to the black hole, whereas they are located somewhat at lower disk radii in wormhole spacetimes. Since each value of $a_{*}$ in the plots is greater than the critical spin parameter (discussed in the next section), the inner edge of the accretion disk, i.e., the left edge of the flux profile, is always located at $r_{0}$, the throat of the wormhole, in contrast to the different positions of the inner edge of the disk around the Kerr black hole.

The different shape functions (35)-(36) in the wormhole metric change only the amplitudes of the flux profiles: the accretion disk of the wormhole with the shape function $b=r_{0}^{2} / r$ produces the radiation flux with highest intensity, and we obtain the lowest values of $\mathcal{F}(r)$ for $b(r)=\left(r_{0} r\right)^{1 / 2}$. By increasing the numerical value of the parameter $\gamma$ in the shape function $b(r)=r_{0}+\gamma r_{0}\left(1-r_{0} / r\right)$, the intensity of the flux is decreasing, as the $1 / r$ term is becoming more and more dominant in $b(r)$.

All these characteristics appear in the disk temperature profiles, depicted in Figs. 3 and 4 as well. The temperature of the disk radiation for the Kerr black hole and the wormhole geometry have the same order of magnitude only for $a_{*} \approx 1$. For lower values of the spin 

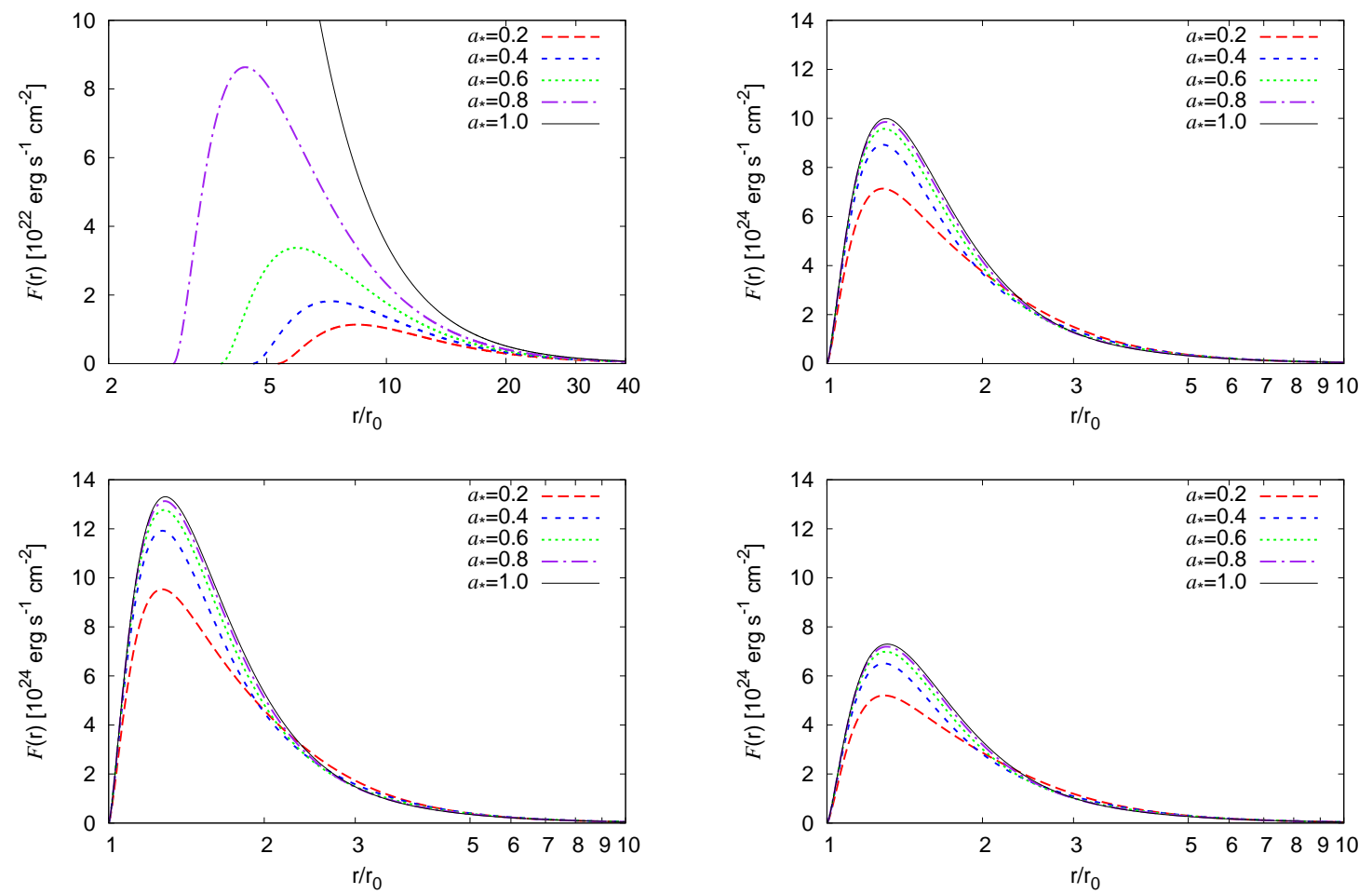

FIG. 1: The energy flux radiated by an accretion disk in a Kerr black hole geometry (upper left hand plot), and in the stationary axially symmetric wormhole spacetimes for $\Phi=-r_{0} / r$ and $b=r_{0}$ (upper right hand plot), $b=r_{0}^{2} / r$ (lower left hand plot) and $b=\left(r_{0} r\right)^{1 / 2}$ (lower right hand plot), respectively. In all plots $r_{0}=1000 \mathrm{~cm}$, and the values of the spin parameter are $a_{*}=0.2,0.4,0.6,0.8$ and 1 , respectively.

parameter the disks rotating around the wormholes are much hotter than those around the Kerr black holes.

In Figs. 5 and [6] we display the disk spectra for the rotating wormholes. Although there are no significant differences between the spectral amplitudes of the disk radiation for the different rotating central objects, the cut-off frequencies of the spectra highly depend on the nature of the holes and their rotational velocities. The cut-off frequencies for the Kerr black hole are systematically lower than those for the wormholes. However, the spectral profiles of the radiation emitted by the accretion disk are rather similar, no matter which of the shape functions $b(r)$ we use for the wormhole spacetimes. The cut-off frequencies of the spectra take the lowest values for $b(r)=\left(r_{0} r\right)^{1 / 2}$, and the highest ones can be found in the plot for $b(r)=r_{0}+\gamma r_{0}\left(1-r_{0} / r\right)$ but these differences are negligible. The plots in Fig. 6 show that the spectral features are also insensitive to the variation of the parameter $\gamma$ in the shape function $b(r)=r_{0}+\gamma r_{0}\left(1-r_{0} / r\right)$.

We also present in Table \ the conversion efficiency $\epsilon$ of the accreting mass into radiation for the case where the photon capture by the Kerr black hole is ignored. The value of $\epsilon$ measures the efficiency of energy generating mechanism by mass accretion. The amount of energy released by matter leaving the accretion disk and falling down the black hole or through the throat of the wormhole is the binding energy $\left.\widetilde{E}(r)\right|_{r=r_{i n}}$ of the hole

\begin{tabular}{|c|cc|cc|}
\hline & \multicolumn{2}{|c|}{ Kerr black hole } & \multicolumn{2}{c|}{ Wormhole } \\
\hline$a_{*}$ & $r_{i n} / r_{0}$ & $\epsilon$ & $r_{i n} / r_{0}$ & $\epsilon$ \\
\hline 0.2 & 5.33 & 0.065 & 1.00 & 0.498 \\
0.4 & 4.62 & 0.075 & 1.00 & 0.506 \\
0.6 & 3.83 & 0.091 & 1.00 & 0.507 \\
0.8 & 2.91 & 0.122 & 1.00 & 0.508 \\
1.0 & 1.00 & 0.421 & 1.00 & 0.508 \\
\hline
\end{tabular}

TABLE I: The inner edge of the accretion disk and the efficiency for rotating black hole and wormhole geometries.

potential.

Table【shows that $\epsilon$ is always higher for rotating wormholes than for Kerr black holes. Even for $a_{*}=0.2$ the conversion efficiency of the accretion process in the wormhole potential is greater than $\epsilon$ derived for the extreme Kerr black holes with $a_{*}=1$. If we consider rapidly rotating wormholes, the efficiency is even higher but there is a limit of $\epsilon$ for high values of the spin parameter: at $a_{*} \gtrsim 0.6$ we obtain $\epsilon=0.508$, where the accretion process is reaching its saturation point, and we cannot attain higher efficiencies even if we increase $a_{*}$ further. However, these high numbers demonstrate that the rotating wormholes provide a much more efficient engine for the 

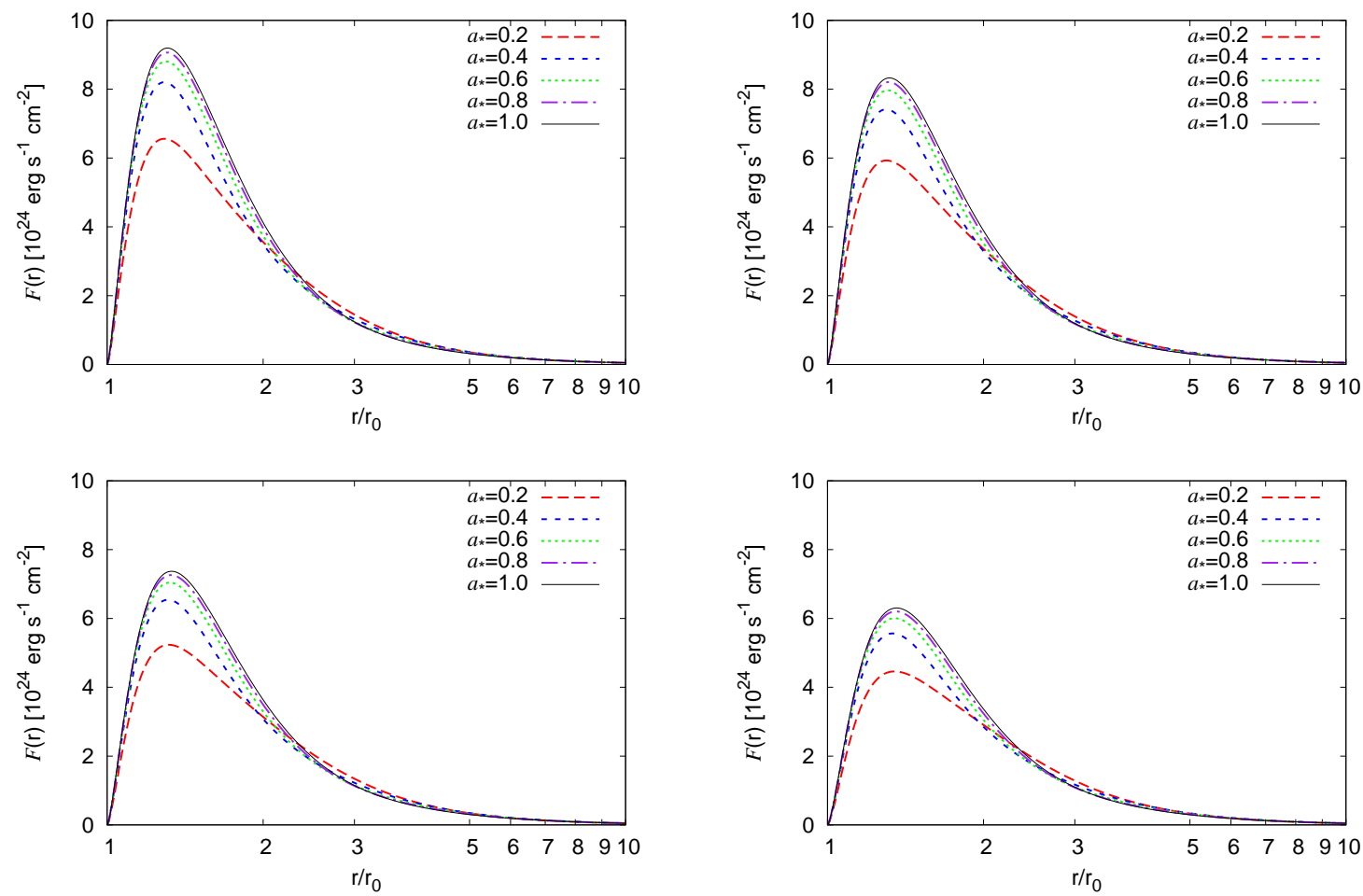

FIG. 2: The energy flux from accretion disks in stationary axially symmetric wormhole spacetimes for $\Phi=-r_{0} / r$, and $b=r_{0}+\gamma r_{0}\left(1-r_{0} / r\right)$, where $\gamma=0.2$ (upper left hand plot), 0.4 (upper right hand plot), 0.6 (lower left hand plot) and 0.8 (lower right hand plot), respectively. In all plots $r_{0}=1000 \mathrm{~cm}$, and the values of the spin parameter are $a_{*}=0.2,0.4,0.6,0.8$ and 1 , respectively.

transformation of the energy of the accreting mass into radiation than the Kerr black holes.

\section{Extremely slowly rotating wormholes}

As seen in the previous section, the inner edge of the accretion disk is always located at $r_{0}$ for any value of the spin parameter we considered. Nevertheless, for extremely small values of $a_{*}$ we found some critical behavior related to the dependence of the location of the marginally stable orbit on the spin parameter. By increasing $a_{*}$ from zero, the radius $r_{m s}$ decreases from $2 r_{0}$. There is a critical value of the spin parameter, namely $a_{*}^{\text {crit }}=0.016693$, where $r_{m s}=1.29 r_{0}$ and any further small rise of $a_{*}$ results in a jump of $r_{m s}$ from $1.29 r_{0}$ to $0.19 r_{0}$. The latter value is already behind the throat of the wormhole, and therefore the boundary of the allowed region for the stable circular orbits $\left(V_{e f f, r r}\right.$ always remains negative) expand from $1.29 r_{0}$ to the throat. This phenomenon is demonstrated in Fig. 7, which depicts the quantity given by Eq. (9) determining the marginally stable orbit and the radial profiles of the photon flux for small values of $a_{*}$.

For a value of the spin parameter between 0 and $a_{*}^{\text {crit }}$ the function $\left(g_{t \phi}^{2}-g_{t t} g_{\phi \phi}\right) V_{e f f, r r}$ has zeros between $2 r_{0}$ and $1.29 r_{0}$. For $a_{*}>a_{*}^{\text {crit }}$ there are no solutions for
Eq. (91), although $\left(g_{t \phi}^{2}-g_{t t} g_{\phi \phi}\right) V_{e f f, r r}<0$ for any $r$ $\left(g_{t \phi}^{2}-g_{t t} g_{\phi \phi}\right.$ is always positive), indicating the presence of stable circular orbits for $r>r_{0}$. At the critical value of the spin parameter the flux profiles also show the jump of the inner edge of the accretion disk from $1.29 r_{0}$ to $r_{0}$.

If we substitute the metric (20) in Eq. (9), we get

$$
\begin{aligned}
0= & V_{e f f, r}=\frac{-2 e^{2 \Phi}\left(\Phi_{, r r}+2 \Phi_{, r}^{2}\right)}{-e^{2 \Phi}+r^{2} K^{2}(\omega-\Omega)^{2}} \\
& +\frac{2 K^{2}\left[\left(\omega+r \omega_{, r}\right)^{2}+r \omega\left(2 \omega_{, r}+r \omega_{, r r}\right)\right]}{-e^{2 \Phi}+r^{2} K^{2}(\omega-\Omega)^{2}} \\
& +\frac{-2 K^{2} \Omega\left[2 \omega+r\left(4 \omega_{, r}+r \omega_{, r r}\right)\right]+2 K^{2} \Omega^{2}}{-e^{2 \Phi}+r^{2} K^{2}(\omega-\Omega)^{2}} \\
& +2\left(K r \omega_{, r} e^{-\Phi}\right)^{2}+8 r^{-1} \Phi_{, r},
\end{aligned}
$$

which is a general formula for any stationary axially symmetric wormhole. Here

$$
\Omega=\frac{1}{2}\left(2 \omega+r \omega_{, r}\right)+\frac{1}{2} \sqrt{r^{2} \omega_{, r}^{2}+4 K^{-2} r^{-1} \Phi, r} e^{2 \Phi} .
$$

For the specific case in study, expression (39) leads to the following relationship

$$
\begin{aligned}
& 0=\frac{e^{-2 / x^{2}}\left[x^{4}+2\left(x^{2}-1\right)\right]+\left(6 a_{*} x^{-2}\right)^{2}}{-x^{4} e^{-2 / x^{2}}+4 a_{*}-\sqrt{9 a_{*}^{2}+x^{6} e^{-2 / x^{2}}}} \\
& +1+\left(6 a_{*} x^{-4} e^{1 / x^{2}}\right)^{2}-4 x^{-2},
\end{aligned}
$$



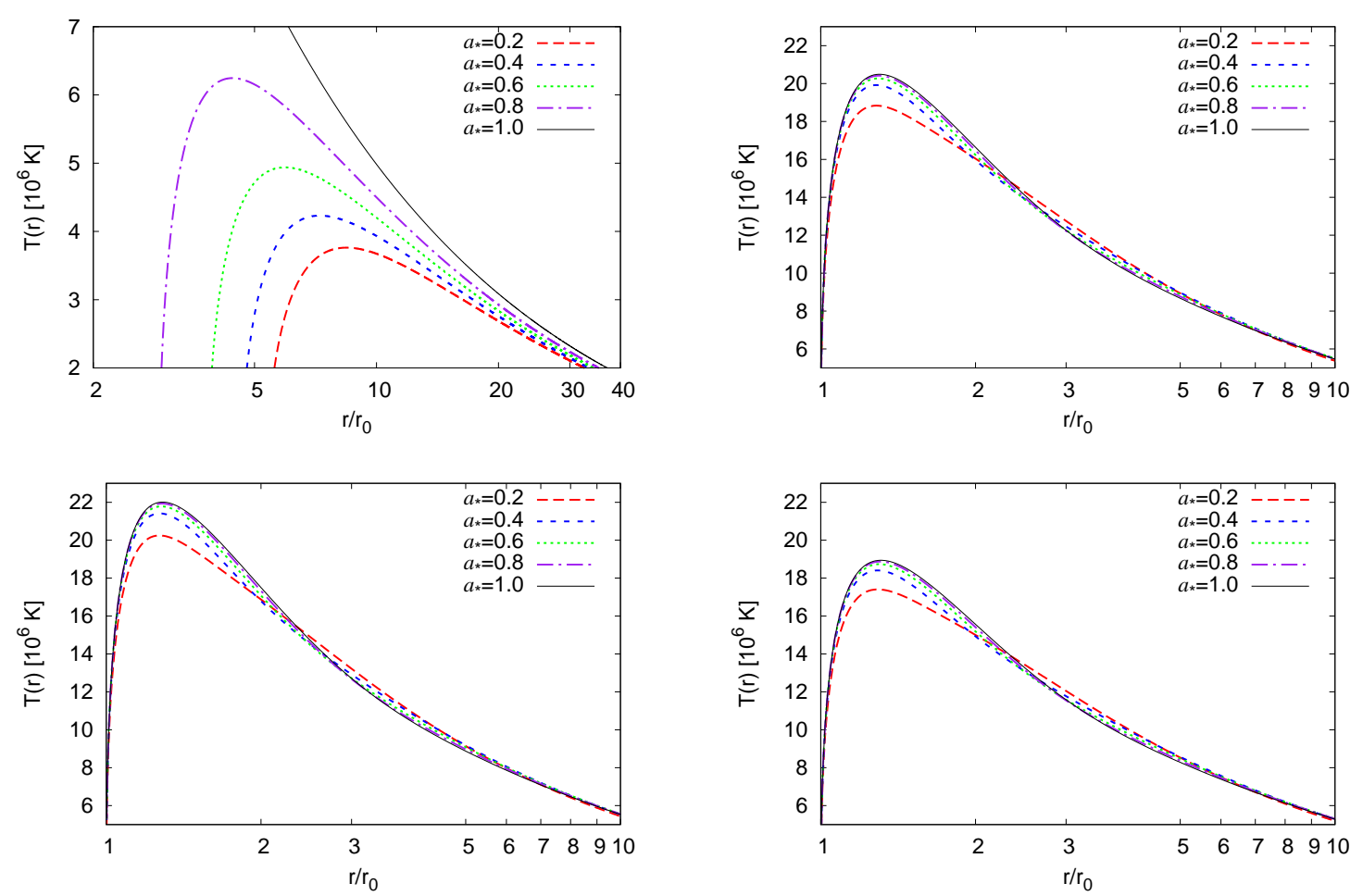

FIG. 3: Temperature distribution of the accretion disk in the Kerr spacetime (upper left hand plot), and of the stationary axially symmetric wormhole spacetimes for $\Phi=-r_{0} / r$, and $b=r_{0}$ (upper right hand plot), $b=r_{0}^{2} / r$ (lower left hand plot), and $b=\left(r_{0} r\right)^{1 / 2}$ (lower right hand plot), respectively. In all plots $r_{0}=1000 \mathrm{~cm}$, and the values of the spin parameter are $a_{*}=0.2,0.4,0.6,0.8$ and 1 , respectively.

where $x^{2}=r / r_{0}$. This result shows that the location of the marginally stable orbit given in the dimensionless radial coordinate $x^{2}$ depends only on $a_{*}$ and the value of $a_{*}^{\text {crit }}$ does not depend on $r_{0}$, as depicted in Fig. 7

By comparing two states with the spin parameters negligibly smaller and greater than $a_{*}^{\text {crit }}$ we see that the distance between the inner edge of the accretion disk and the wormhole throat is $1.29 r_{0}-r_{0}=0.29 r_{0}$ in both cases. If the accretion process increases the spin of the wormhole such that it just exceeds $a_{*}^{\text {crit }}$ with a infinitesimally small value then the inner edge of the disk will fall into the throat with supersonic velocity, eliminating the so called plunging region located between the disk and the rotating central object. It must have a strong physical influence, e.g., shock waves are generated. However, after this transient situation, the inner edge of the disk will be located at $r_{0}$ and all the orbits for $r>r_{0}$ will be stable $\left(V_{e f f, r r}<0\right)$, i.e., the thin accretion disk exists in the whole equatorial plane outside the throat.

\section{DISCUSSIONS AND FINAL REMARKS}

In the present paper, we have studied thin accretion disk models in stationary axially symmetric wormhole geometries, and have carried out an analysis of the properties of the radiation emerging from the surface of the disk. In classical general relativity, wormholes are supported by exotic matter, which involves a stress-energy tensor that violates the null energy condition. Thus, it is important to analyze the properties of the accretion disks around wormholes supported by exotic matter, namely, the time averaged energy flux, the disk temperature and the emission spectra. By comparing the accretion disk properties in a stationary rotating wormhole geometry with the properties of disks around a Kerr black hole, we have shown that the intensity of the flux emerging from the disk surface is greater for wormholes than for the rotating black hole with the same geometrical mass $r_{0}$ and accretion rate $\dot{M}_{0}$. We also presented the conversion efficiency $\epsilon$ of the accreting mass into radiation, and proved that rotating wormholes are much more efficient in converting the accreting mass into radiation than the Kerr black holes.

It is generally expected that most of the astrophysical objects grow substantially in mass via accretion. Recent observations suggest that around most of the active galactic nuclei (AGN's) or black hole candidates there exist gas clouds surrounding the central far object, and an associated accretion disk, on a variety of scales from a tenth of a parsec to a few hundred parsecs [17]. These clouds are assumed to form a geometrically and optically thick torus (or warped disk), which absorbs most of the ultraviolet radiation and the soft x-rays. The gas 

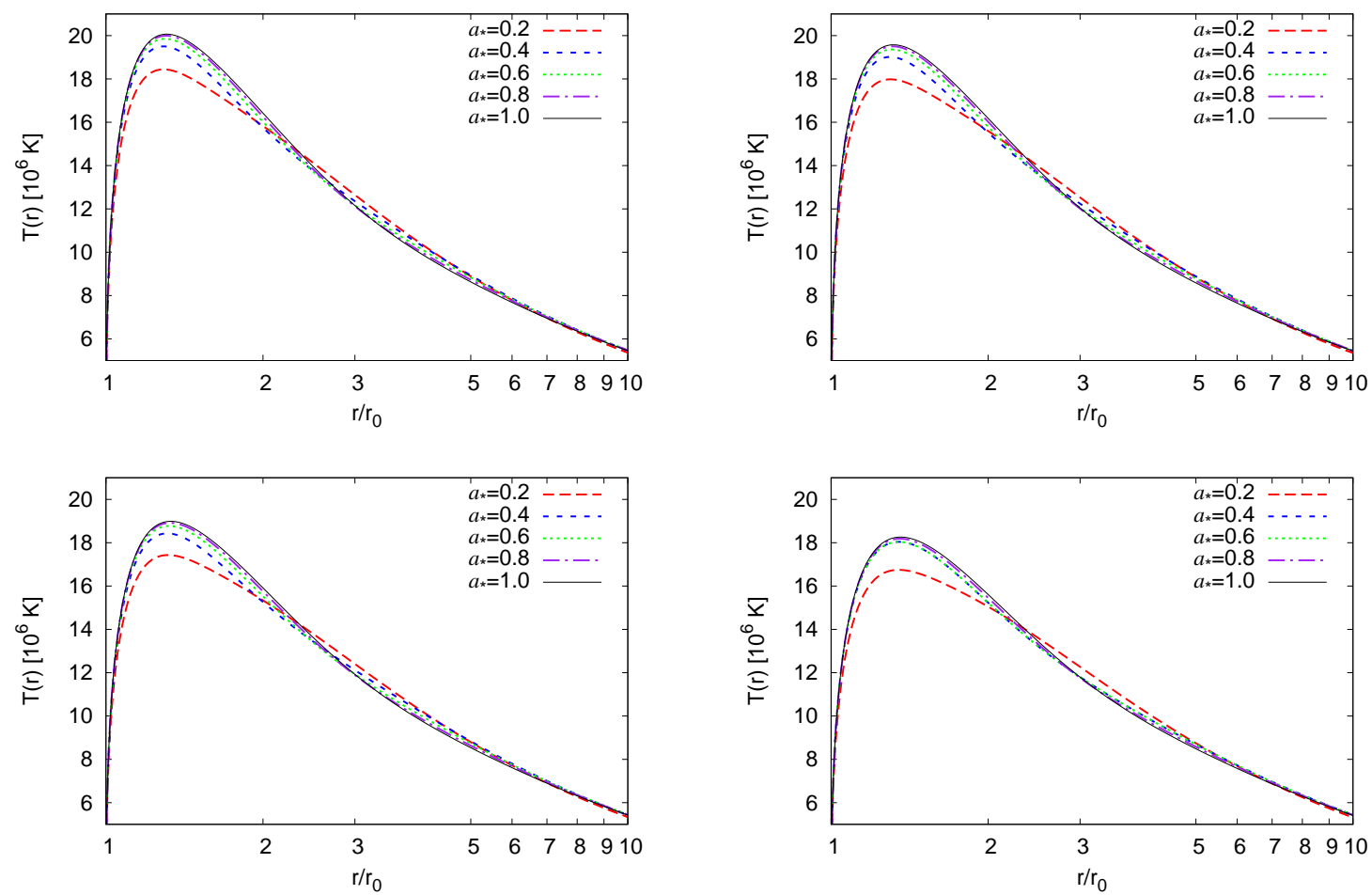

FIG. 4: Temperature distribution of the accretion disk in the stationary axially symmetric wormhole spacetime for $\Phi=-r_{0} / r$, and $b=r_{0}+\gamma r_{0}\left(1-r_{0} / r\right)$, where $\gamma=0.2$ (upper left hand plot), 0.4 (upper right hand plot), 0.6 (lower left hand plot) and 0.8 (lower right hand plot), respectively. In all plots $r_{0}=1000 \mathrm{~cm}$, and the values of the spin parameter are $a_{*}=0.2,0.4,0.6,0.8$ and 1 , respectively.

exists in either the molecular or the atomic phase. The most powerful evidence for the existence of super massive black holes comes from the very long baseline interferometry (VLBI) imaging of molecular $\mathrm{H}_{2} \mathrm{O}$ masers in the active galaxy NGC 4258 [18]. This imaging, produced by Doppler shift measurements assuming Keplerian motion of the masering source, has allowed a quite accurate estimation of the central mass, which has been found to be a $3.6 \times 10^{7} M_{\odot}$ super massive dark object, within 0.13 parsecs. Hence, important astrophysical information can be obtained from the observation of the motion of the gas streams in the gravitational field of compact objects.

The determination of the accretion rate for an astrophysical object can give a strong evidence for the existence of a surface of the object. A model in which Sgr A*, the $3.7 \times 10^{6} M_{\odot}$ super massive black hole candidate at the Galactic center, may be a compact object with a thermally emitting surface was considered in [19]. For very compact surfaces within the photon orbit, the thermal assumption is likely to be a good approximation because of the large number of rays that are strongly gravitationally lensed back onto the surface. Given the very low quiescent luminosity of Sgr $\mathrm{A}^{*}$ in the near-infrared, the existence of a hard surface, even in the limit in which the radius approaches the horizon, places a severe constraint on the steady mass accretion rate onto the source, $\dot{M} \leq 10^{-12} M_{\odot} \mathrm{yr}^{-1}$. This limit is well below the mini- mum accretion rate needed to power the observed submillimeter luminosity of $\mathrm{Sgr} \mathrm{A}^{*}, \dot{M} \geq 10^{-10} M_{\odot}$ yr. Thus, from the determination of the accretion rate it follows that Sgr A* does not have a surface, that is, it must have an event horizon. Therefore the study of the accretion processes by compact objects is a powerful indicator of their physical nature. However, up to now, the observational results have confirmed the predictions of general relativity mainly in a qualitative way. With the present observational precision one cannot distinguish between the different classes of compact/exotic objects that appear in the theoretical framework of general relativity [4].

However, important technological developments may allow one to image black holes and other compact objects directly [19]. For a black hole embedded in an accretion flow, the silhouette will generally be asymmetric regardless of the spin of the black hole. Even in an optically thin accretion flow an asymmetry will result from special relativistic effects (aberration and Doppler shifting). In principle, detailed measurements of the size and shape of the silhouette could yield information about the mass and spin of the central object, and provide invaluable information on the nature of the accretion flows in low luminosity galactic nuclei. With the improvement of the imaging observational techniques, which give the physi$\mathrm{cal} /$ geometrical properties of the silhouette of the com- 

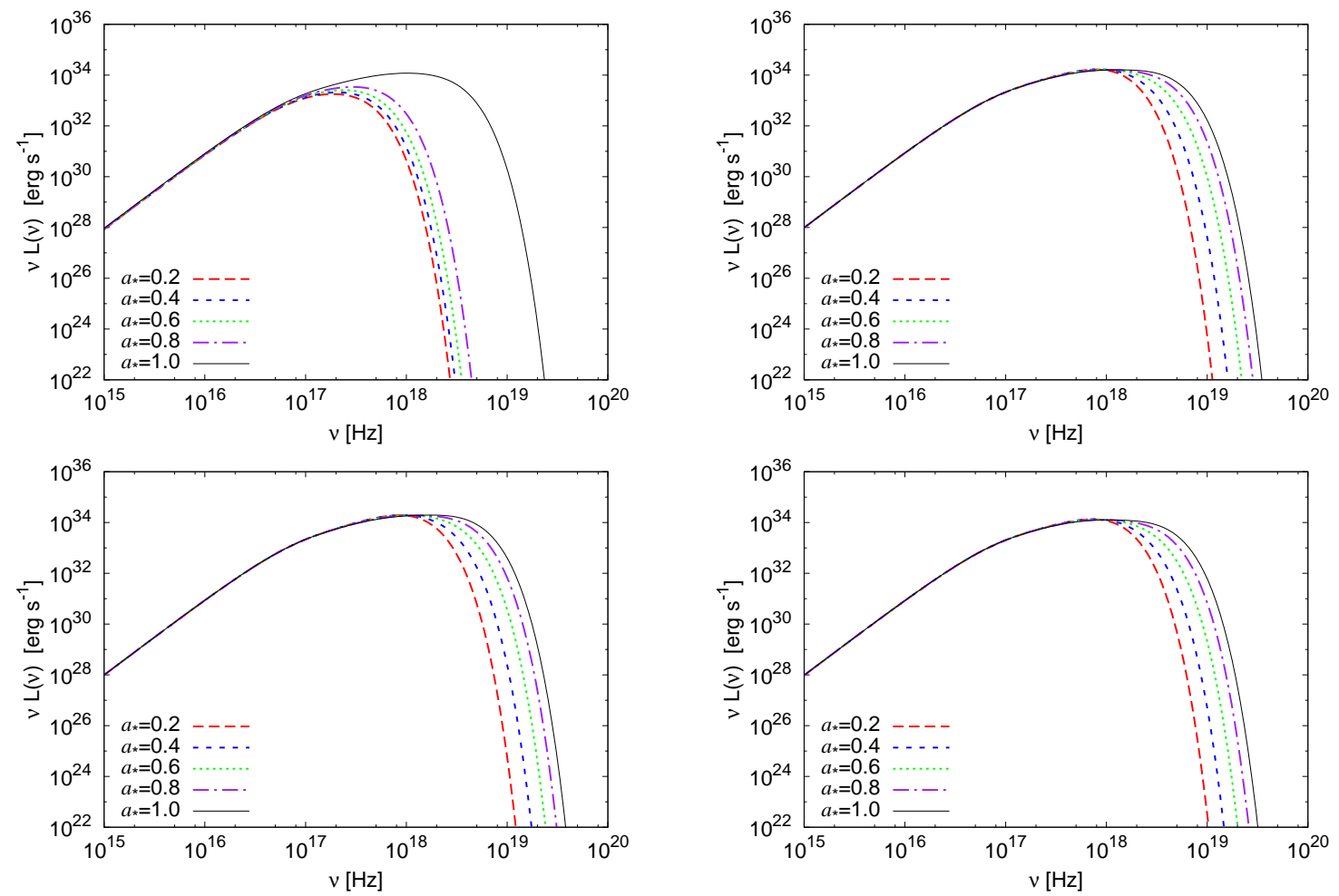

FIG. 5: The emission spectra of the accretion disk in the Kerr spacetime (upper left hand plot) and in the stationary axially symmetric wormhole spacetime for $\Phi=-r_{0} / r$, and $b=r_{0}$ (upper right hand plot), $b=r_{0}^{2} / r$ (lower left hand plot), and $b=\left(r_{0} r\right)^{1 / 2}$ (lower right hand plot), respectively. In all plots $r_{0}=1000 \mathrm{~cm}$, and the values of the spin parameter are $a_{*}=0.2,0.4,0.6,0.8$ and 1 , respectively.

pact object cast upon the accretion flows, it will also be possible to provide clear observational evidence for the existence of wormholes, and to differentiate them from other types of compact general relativistic objects.

In this context, we conclude our study by pointing out that the specific properties that appear in the physical characteristics of the thin accretion disks around wormholes can lead to the possibility of directly detecting and discriminating wormhole geometries by observing accretion disks around compact astrophysical objects.

\section{Acknowledgments}

The work of $\mathrm{TH}$ is supported by an RGC grant of the government of the Hong Kong SAR. ZK was supported by the Hungarian Scientific Research Fund (OTKA) grant no.69036. FSNL was partially funded by Fundação para a Ciência e a Tecnologia (FCT)-Portugal through the grant SFRH/BPD/26269/2006.

\section{APPENDIX A: EFFECTIVE POTENTIAL FOR THE KERR BLACK HOLE}

The Kerr metric in the Boyer-Lindquist (BL) coordinate system is given by

$$
\begin{aligned}
d s^{2} & =-\left(1-\frac{2 m r}{\Sigma}\right) d t^{2}+2 \frac{2 m r}{\Sigma} a \sin ^{2} \theta d t d \phi+\frac{\Sigma}{\Delta} d r^{2} \\
& +\Sigma d \theta^{2}+\left(r^{2}+a^{2}+\frac{2 m r}{\Sigma} a^{2} \sin ^{2} \theta\right) \sin ^{2} \theta d \phi^{2} .(\mathrm{A} 1)
\end{aligned}
$$

In the equatorial plane, the metric components reduce to

$$
\begin{aligned}
g_{t t} & =-\left(1-\frac{2 m r}{\Sigma}\right)=-\left(1-\frac{2 m}{r}\right) \\
g_{t \phi} & =\frac{2 m r}{\Sigma} a \sin ^{2} \theta=2 \frac{m a}{r} \\
g_{r r} & =\frac{\Sigma}{\Delta}=\frac{r^{2}}{\Delta} \\
g_{\phi \phi} & =\left(r^{2}+a^{2}+\frac{2 m r}{\Sigma} a^{2} \sin ^{2} \theta\right) \sin ^{2} \theta \\
& =r^{2}+a^{2}\left(1+\frac{2 m}{r}\right),
\end{aligned}
$$

respectively. 

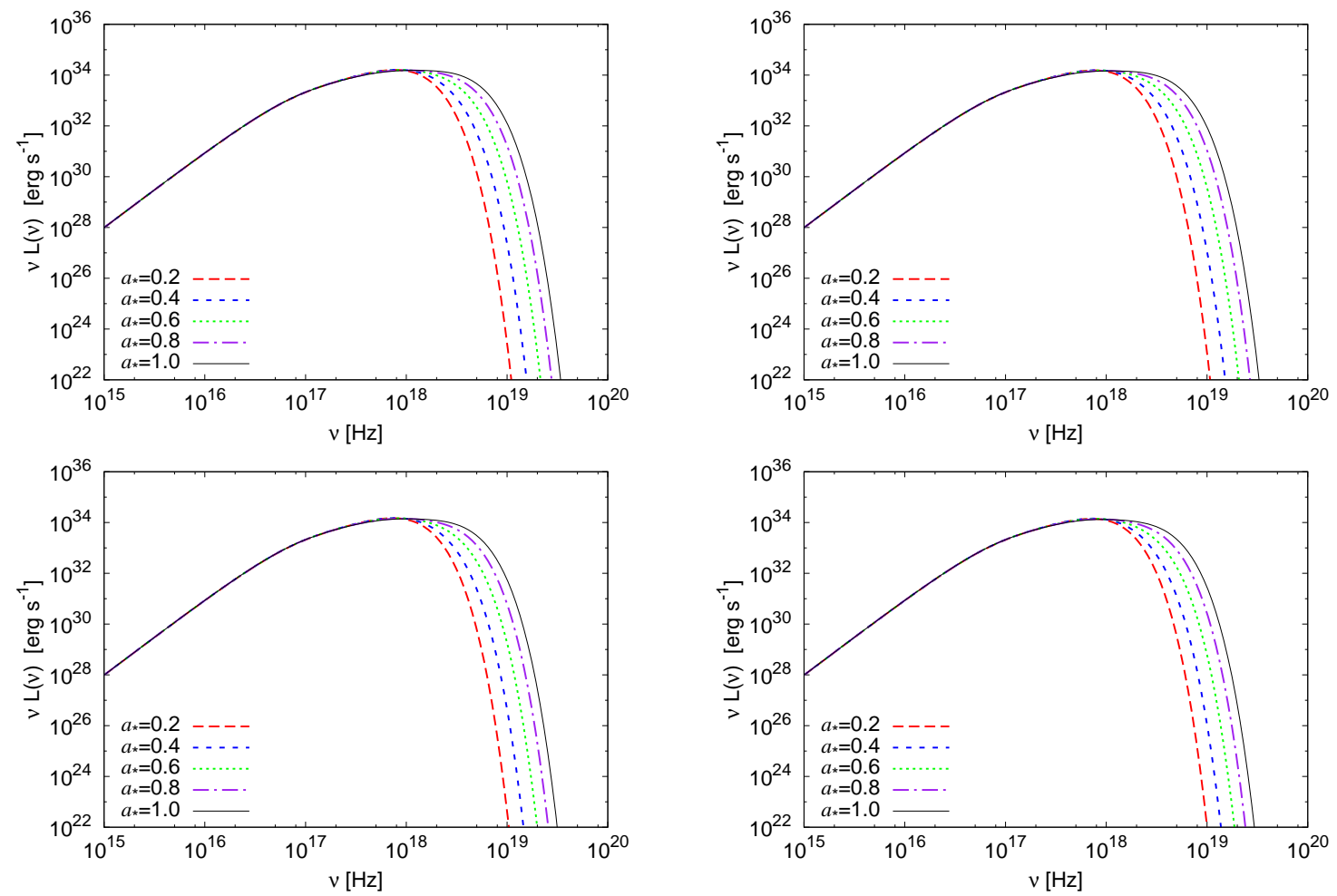

FIG. 6: The emission spectra of the accretion disk in the stationary axially symmetric wormhole spacetime for $\Phi=-r_{0} / r$, and $b=r_{0}+\gamma r_{0}\left(1-r_{0} / r\right)$, where $\gamma=0.2$ (upper left hand plot), 0.4 (upper right hand plot), 0.6 (lower left hand plot) and 0.8 (lower right hand plot), respectively. In all plots $r_{0}=1000 \mathrm{~cm}$, and the values of the spin parameter are $a_{*}=0.2,0.4,0.6,0.8$ and 1 , respectively.
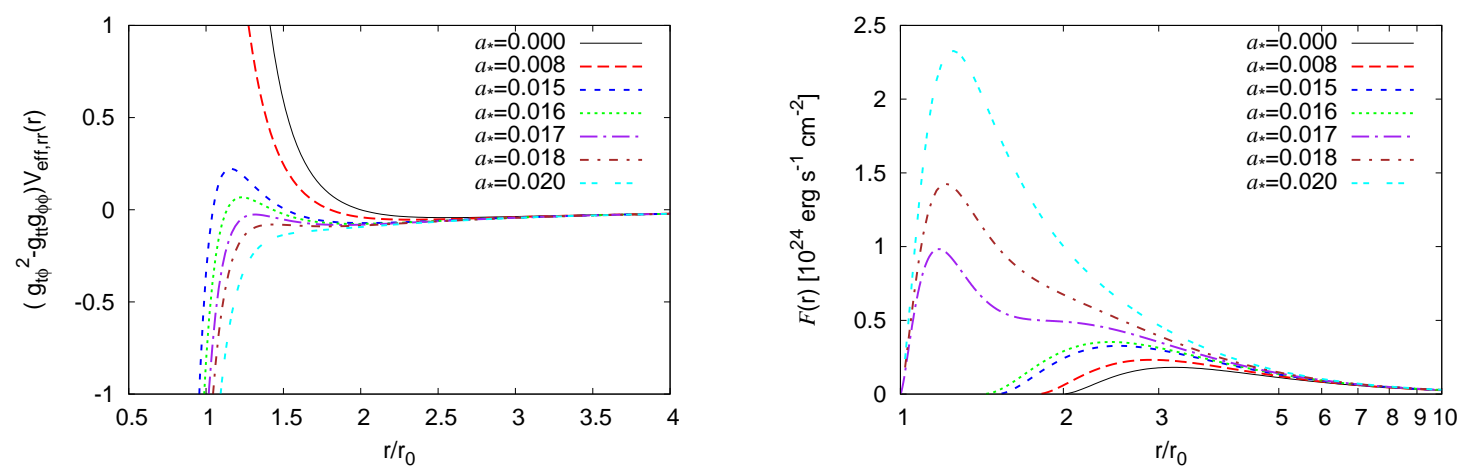

FIG. 7: The function $\left(g_{t \phi}^{2}-g_{t t} g_{\phi \phi}\right) V_{e f f, r r}$ vs. $r / r_{0}$ (left hand plot) and the flux radiated by the accretion disk (right hand plot) in the stationary axially symmetric wormhole spacetime for $\Phi=-r_{0} / r$, and $b=r_{0}$ for extremely low values of the spin parameter between 0 and 0.02 .

The geodesic equation (4) for $r$ is

$$
\frac{r^{2}}{\Delta}\left(\frac{d r}{d \tau}\right)^{2}=V_{e f f}(r)
$$

with the effective potential given by

$$
\begin{array}{r}
V_{\text {eff }}(r)=-1+\left\{\widetilde{E}^{2}\left[r^{2}\left(r^{2}+a^{2}\right)+2 m a^{2} r\right]\right. \\
\left.+4 \widetilde{E} \widetilde{L} m a r-\widetilde{L}^{2}\left(r^{2}-2 m r\right)\right\} /\left[r^{2}\left(g_{t \phi}^{2}-g_{t t} g_{\phi \phi}\right)\right] .
\end{array}
$$

Note that these relationships may be rewritten as

$$
r^{4}\left(\frac{d r}{d \tau}\right)^{2}=V(r)
$$

with $V(r)$ given by

$$
V(r)=r^{2} \Delta V_{e f f}(r)=r^{2}\left(g_{t \phi}^{2}-g_{t t} g_{\phi \phi}\right) V_{e f f}(r),
$$

where the relationship $\Delta=g_{t \phi}^{2}-g_{t t} g_{\phi \phi}=r^{2}-2 m r+a^{2}$ along the equatorial plane has been used. 
[1] T. Harko, Z. Kovacs and F. S. N. Lobo, Phys. Rev. D 78, 084005 (2008) arXiv:0808.3306 [gr-qc]].

[2] S. Bhattacharyya, A. V. Thampan and I. Bombaci, Astron. Astrophys. 372, 925 (2001).

[3] D. Torres, Nucl. Phys. B 626, 377 (2002).

[4] Y. F. Yuan, R. Narayan and M. J. Rees, Astrophys. J. 606, 1112 (2004).

[5] F. S. Guzman, Phys. Rev. D 73, 021501 (2006).

[6] C. S. J. Pun, Z. Kovacs and T. Harko, Phys. Rev. D 78, 024043 (2008).

[7] C. S. J. Pun, Z. Kovacs and T. Harko, Phys. Rev. D 78, 084015 (2008).

[8] I. D. Novikov and K. S. Thorne, in Black Holes, ed. C. DeWitt and B. DeWitt, New York: Gordon and Breach (1973).

[9] N. I. Shakura and R. A. Sunyaev, Astron. Astrophys. 24, 33 (1973).

[10] D. N. Page and K. S. Thorne, Astrophys. J. 191, 499 (1974).

[11] K. S. Thorne, Astrophys. J. 191, 507 (1974).

[12] M. S. Morris and K. S. Thorne, Am. J. Phys. 56, 395 (1988); H. G. Ellis, J. Math. Phys. 14 (1973) 104; K. A. Bronnikov, Acta Phys. Polon. B 4 (1973) 251; G. Clement, Gen. Rel. Grav. 16, 131 (1984); G. Clement, Gen. Rel. Grav. 16, 477 (1984); G. Clement, Gen. Rel. Grav. 16, 491 (1984); B. Bhawal and S. Kar, Phys. Rev. D 46, 2464-2468 (1992); G. Dotti, J. Oliva, and R. Troncoso, Phys. Rev. D 75, 024002 (2007); L. A. Anchordoqui and S. E. P. Bergliaffa, Phys. Rev. D 62, 067502 (2000); K. A. Bronnikov and S.-W. Kim, Phys. Rev. D 67, 064027 (2003); M. La Camera, Phys. Lett. B573, 27-32 (2003); F. S. N. Lobo, Phys. Rev. D 75, 064027 (2007); K. K. Nandi, B. Bhattacharjee, S. M. K. Alam and J. Evans, Phys. Rev.
D 57, 823 (1998); R. Garattini and F. S. N. Lobo, Class. Quant. Grav. 24, 2401 (2007); R. Garattini and F. S. N. Lobo, Phys. Lett. B 671, 146 (2009); C. G. Boehmer, T. Harko and F. S. N. Lobo, Phys. Rev. D 76, 084014 (2007); C. G. Boehmer, T. Harko and F. S. N. Lobo, Class. Quant. Grav. 25, 075016 (2008); A. DeBenedictis, R. Garattini and F. S. N. Lobo, Phys. Rev. D 78, 104003 (2008); S. Sushkov, Phys. Rev. D 71, 043520 (2005); F. S. N. Lobo, Phys. Rev. D 71, 084011 (2005); F. S. N. Lobo, Phys. Rev. D 71, 124022 (2005); F. S. N. Lobo, Phys. Rev. D 73, 064028 (2006); F. S. N. Lobo, Phys. Rev. D 75, 024023 (2007); F. S. N. Lobo, Class. Quant. Grav. 25, 175006 (2008); J. P. S. Lemos, F. S. N. Lobo and S. Quinet de Oliveira, Phys. Rev. D 68, 064004 (2003); J. P. S. Lemos and F. S. N. Lobo, Phys. Rev. D 69, 104007 (2004); H. Maeda and M. Nozawa, Phys. Rev. D 78, 024005 (2008); H. Maeda, to appear in Physical Review D, arXiv:0811.2962 [gr-qc]; H. Maeda, T. Harada and B. J. Carr, arXiv:0901.1153 [gr-qc].

[13] F. S. N. Lobo, arXiv:0710.4474 [gr-qc].

[14] E. Teo, Phys. Rev. D 58, 024014 (1998).

[15] J. P. Luminet, Astron. Astrophys. 75, 228 (1979).

[16] S. Bhattacharyya, R. Misra, and A. V. Thampan, Astrophys. J. 550, 841 (2001).

[17] C. M. Urry and P. Padovani, Publ. Astron. Soc. of the Pacific 107, 803 (1995).

[18] M. Miyoshi, J. Moran, J. Herrnstein, L. Greenhill, N. Nakai, P. Diamond and M. Inoue, Nature 373, 127 (1995).

[19] A. E. Broderick and R. Narayan, Astrophys. JL. 636, L109 (2006). 